PROCEEDINGS OF THE AMERICAN MATHEMATICAL SOCIETY

Volume 124, Number 1, January 1996

\title{
INNER INVARIANT MEANS AND CONJUGATION OPERATORS
}

\author{
YUJI TAKAHASHI
}

(Communicated by J. Marshall Ash)

Dedicated to Professor Satoru Igari on his sixtieth birthday

\begin{abstract}
It is shown that Paschke's result cannot be generalized to the [IN]group setting given by Lau and Paterson. This resolves negatively a problem raised by Lau and Paterson.
\end{abstract}

Let $G$ be an [IN]-group with a fixed Haar measure $\lambda$, and let $L^{p}(G)(1 \leq p \leq \infty)$ be the associated Lebesgue spaces. Recall that a locally compact group $G$ is said to be an $[I N]$-group if $G$ contains a compact neighborhood of the identity in $G$ which is invariant under all inner automorphisms. Notice that an [IN]-group is unimodular. It is also well known that $L^{\infty}(G)$ has an inner invariant mean $m$, i.e., a mean on $L^{\infty}(G)$ such that $m(\pi(a) f)=m(f)$ for all $a \in G$ and $f \in L^{\infty}(G)$, where $\pi(a) f(x)=f\left(a^{-1} x a\right)(x \in G)$. (A locally compact group $G$ is called inner amenable if $L^{\infty}(G)$ admits an inner invariant mean. Some results on inner amenability are to be found in $[1,3,4,5,6,7,10]$.) For a compact neighborhood $V$ of the identity in $G$, let

$$
L_{0}^{2}(V)=\left\{g \in L^{2}(G): \int_{V} g(x) d \lambda(x)=0\right\}
$$

and let $P_{V}$ denote the orthogonal projection on the one-dimensional subspace of $L^{2}(G)$ spanned by $\mathbf{1}_{V}$ (the characteristic function of $V$ ). Thus the operator $P_{V}$ has the form

$$
P_{V}(f)=\left(\frac{1}{\lambda(V)} \int_{V} f(x) d \lambda(x)\right) \cdot \mathbf{1}_{V} \quad\left(f \in L^{2}(G)\right) .
$$

For $p \in[1, \infty)$, let $\pi_{p}$ be the isometric representation of $G$ on $L^{p}(G)$ defined by

$$
\pi_{p}(x) f(t)=f\left(x^{-1} t x\right) \quad\left(x, t \in G, f \in L^{p}(G)\right) .
$$

Let $C^{*}\left(\pi_{2}(G)\right)$ denote the $C^{*}$-algebra generated by $\pi_{2}(G)$ in $\mathcal{B}\left(L^{2}(G)\right)$ (the space of bounded linear operators on $L^{2}(G)$ ).

For a compact neighborhood $V$ of the identity in $G$ which is invariant under all inner automorphisms, let us consider the following conditions on $G$ :

(a) $P_{V}$ is not in $C^{*}\left(\pi_{2}(G)\right)$.

Received by the editors July 29, 1994.

1991 Mathematics Subject Classification. Primary 43A07, 43A15.

Key words and phrases. [IN]-groups, inner invariant means, amenability, free groups, $C^{*}-$ algebras. 
(b) There exists a net $\left\{h_{\alpha}\right\}$ in $L_{0}^{2}(V)$ such that $\left\|h_{\alpha}\right\|_{2}=1$ and $\left\|\pi_{2}(x) h_{\alpha}-h_{\alpha}\right\|_{2} \rightarrow$ 0 for each $x \in G$.

(c) There exists a state $\omega$ on $\mathcal{B}\left(L^{2}(G)\right)$ such that $\omega\left(\pi_{2}(x)\right)=1$ for each $x \in G$ and $\omega\left(P_{V}\right)=0$.

(d) There exists an inner invariant mean $m$ on $L^{\infty}(G)$ such that $m\left(\mathbf{1}_{V}\right)=0$.

In [4, Theorem 4.2] Lau and Paterson proved that (a), (b), and (c) are equivalent and that (d) implies one (and hence all) of (a), (b), and (c). The following was posed as an open problem in [4, p. 164]: Is (d) equivalent to the other conditions? Note that this is the case when $G$ is an infinite discrete group and $V=\{e\}$ (where $e$ is the identity of $G$ ) as Paschke's result [7] shows. Notice also that the answer to the Lau-Paterson problem is obviously negative if $G$ is a compact abelian group and $V=G$. Indeed, since $G$ is abelian, $\pi_{2}(x) h=h$ for each $x \in G$ and $h \in L_{0}^{2}(G)$ and therefore (b) holds. However, (d) is not true because $m\left(\mathbf{1}_{G}\right)=1$ for any mean $m$ on $L^{\infty}(G)$. The main purpose of this note is to give an example which shows that the answer to the Lau-Paterson problem is negative even though $V \neq G$. We also prove that if $G$ is amenable and $V \neq G$, then (d) (and hence any one of (a), (b), and (c)) holds. (For more information on amenable groups, see [2, 8, 9].)

Let us begin with the following example which gives the negative answer to the Lau-Paterson problem.

Example 1. Let $G$ be the direct product of a finite abelian group $H$ of order $n \geq 2$ and the free group $\mathbf{F}_{2}$ on two generators, and let $V=H \times\{e\}$ (where $e$ denotes the identity of $\mathbf{F}_{2}$ ). Of course, $V$ is a compact neighborhood of the identity in $G$ which is invariant under all inner automorphisms. We shall prove that $G$ satisfies (b) but not (d). It is easy to verify that $G$ meets (b). Indeed, take a function $g \in l_{0}^{2}(H)\left(=L_{0}^{2}(H)\right)$ and consider the function $h$ on $G$ defined by

$$
h((s, t))= \begin{cases}g(s) & \text { if }(s, t) \in V(=H \times\{e\}), \\ 0 & \text { otherwise }\end{cases}
$$

Then, since $h \in l_{0}^{2}(V)$ and $\pi_{2}((x, y)) h-h=0$ for each $(x, y) \in G\left(=H \times \mathbf{F}_{2}\right)$, (b) holds. Let us next show that $G$ does not satisfy (d). Assume, by way of contradiction, that there exists an inner invariant mean $m$ on $l^{\infty}(G)\left(=L^{\infty}(G)\right)$ such that $m\left(\mathbf{1}_{V}\right)=0$. Denote two generators of $\mathbf{F}_{2}$ by $a$ and $b$, and let $A$ be the subset of $\mathbf{F}_{2}$ formed by all reduced words of the form $\ldots a^{n}$ where $n$ is a nonzero integer. Note that $\mathbf{F}_{2}=A \cup a A a^{-1} \cup\{e\}$ and the subsets $A, b A b^{-1}$, and $b^{-1} A b$ are mutually disjoint. Let $H=\left\{s_{1}, s_{2}, \ldots, s_{n}\right\}$ where $s_{1}=0$ (the identity of $H$ ), and put $B_{i}=\left\{s_{i}\right\} \times A(1 \leq i \leq n)$. Then

$$
\begin{aligned}
G & =H \times \mathbf{F}_{2}=H \times\left(A \cup a A a^{-1} \cup\{e\}\right) \\
& =\left(\bigcup_{i=1}^{n} B_{i}\right) \cup\left(\bigcup_{i=1}^{n}{ }_{\xi}\left(B_{i}\right)_{\xi^{-1}}\right) \cup V,
\end{aligned}
$$

where $\xi=(0, a)$. Hence we have

$$
\begin{aligned}
2 \sum_{i=1}^{n} m\left(\mathbf{1}_{B_{i}}\right) & =\sum_{i=1}^{n} m\left(\mathbf{1}_{B_{i}}\right)+\sum_{i=1}^{n} m\left(\pi(\xi) \mathbf{1}_{B_{i}}\right)+m\left(\mathbf{1}_{V}\right) \\
& \geq m\left(\mathbf{1}_{G}\right)=1
\end{aligned}
$$


because $m\left(\mathbf{1}_{V}\right)=0$ and $m$ is inner invariant. On the other hand, since the subsets $B_{i},{ }_{\eta}\left(B_{i}\right)_{\eta^{-1}}$, and $\eta^{-1}\left(B_{i}\right)_{\eta}(1 \leq i \leq n)$ are mutually disjoint for $\eta=(0, b)$,

$$
\begin{aligned}
1 & =m\left(\mathbf{1}_{G}\right) \geq \sum_{i=1}^{n} m\left(\mathbf{1}_{B_{i}}\right)+\sum_{i=1}^{n} m\left(\pi(\eta) \mathbf{1}_{B_{i}}\right)+\sum_{i=1}^{n} m\left(\pi\left(\eta^{-1}\right) \mathbf{1}_{B_{i}}\right) \\
& =3 \sum_{i=1}^{n} m\left(\mathbf{1}_{B_{i}}\right) .
\end{aligned}
$$

But this gives the desired contradiction. Thus we conclude that $G$ does not satisfy (d).

Remarks. (1) The group $G$ in Example 1 is not amenable. In fact, as shown below (Proposition 3), it is impossible to find a counterexample to the Lau-Paterson problem for amenable [IN]-groups.

(2) The argument used in Example 1 shows that if $G$ is the direct product of $\mathbf{F}_{2}$ and a finite (not necessarily abelian) group of order greater than or equal to 2 , then $G$ does not satisfy (d).

We now proceed to a characterization of [IN]-group for which (d) holds.

Proposition 2. Let $G$ be an $[I N]$-group, and let $V$ be a compact neighborhood of the identity in $G$ which is invariant under all inner automorphisms. Let $1 \leq p<\infty$. Then the following assertions are equivalent:

(i) There exists a net $\left\{k_{\alpha}\right\}$ in $L^{p}(G)$ such that $k_{\alpha} \geq 0, k_{\alpha} \mid V=0,\left\|k_{\alpha}\right\|_{p}=1$, and $\left\|\pi_{p}(x) k_{\alpha}-k_{\alpha}\right\|_{p} \rightarrow 0$ for each $x \in G$.

(ii) $L^{\infty}(G)$ admits an inner invariant mean $m$ such that $m\left(\mathbf{1}_{V}\right)=0$.

Proof. First of all, note that it suffices to show the case $p=1$. Indeed, this follows immediately from the inequality

$$
\left(\left\|f^{1 / p}-g^{1 / p}\right\|_{p}\right)^{p} \leq\|f-g\|_{1} \leq p 2^{p-1}\left\|f^{1 / p}-g^{1 / p}\right\|_{p}
$$

$\left(f, g \in\left\{h \in L^{1}(G): h \geq 0,\|h\|_{1}=1\right\}\right.$ and $p \in[1, \infty)$ ). Let us suppose that $p=1$ in the remainder of the proof.

Let $\left\{k_{\alpha}\right\} \subset L^{1}(G) \subset L^{\infty}(G)^{*}$ be given as in (i), and let $m$ be any weak*-cluster point of $\left\{k_{\alpha}\right\}$ in $L^{\infty}(G)^{*}$. We may assume by passing to a subnet if necessary that $\left\{k_{\alpha}\right\}$ converges to $m$ in the weak*-topology in $L^{\infty}(G)^{*}$. Since each $k_{\alpha}$ is a mean on $L^{\infty}(G)$ and $\left\|\pi_{1}(x) k_{\alpha}-k_{\alpha}\right\|_{1} \rightarrow 0$ for each $x \in G, m$ is an inner invariant mean on $L^{\infty}(G)$. Moreover, we have

$$
m\left(\mathbf{1}_{V}\right)=\lim _{\alpha} k_{\alpha}\left(\mathbf{1}_{V}\right)=0
$$

because $k_{\alpha} \mid V=0$. Thus (i) implies (ii).

Conversely, let $m$ be an inner invariant mean on $L^{\infty}(G)$ such that $m\left(\mathbf{1}_{V}\right)=0$. Then, from a well-known argument (cf. the proof of [6, Proposition 1]) and the fact that $m\left(\mathbf{1}_{V}\right)=0$, it is easy to find a net $\left\{k_{\alpha}\right\}$ in $L^{1}(G)$ such that $k_{\alpha} \geq 0, k_{\alpha} \mid V=$ $0,\left\|k_{\alpha}\right\|_{1}=1$, and $\left\|\pi_{1}(x) k_{\alpha}-k_{\alpha}\right\|_{1} \rightarrow 0$ for each $x \in G$. This completes the proof.

The following proposition can be combined with Lau-Paterson's result [4, Theorem 4.2] to show that (a), (b), (c), and (d) are equivalent if $G$ is amenable.

Proposition 3. Let $G$ be an amenable $[I N]$-group , and let $V$ be a compact neighborhood of the identity in $G$ which is invariant under all inner automorphisms. If $V \neq G$, then $L^{\infty}(G)$ has an inner invariant mean $m$ such that $m\left(\mathbf{1}_{V}\right)=0$. 
Proof. If $G$ is noncompact, then $m\left(\mathbf{1}_{V}\right)=0$ for any two-sided invariant mean $m$ on $L^{\infty}(G)$ (cf. [9, Proposition 21.2]). Since a two-sided invariant mean on $L^{\infty}(G)$ is inner invariant, there exists the desired inner invariant mean. Assume next that $G$ is compact. Let $h=\frac{1}{\lambda\left(V^{c}\right)} \mathbf{1}_{V} c$; then $h \geq 0, h \mid V=0,\|h\|_{1}=1$, and $\pi_{1}(x) h-h=0$ for each $x \in G$. Hence an application of Proposition 2 yields an inner invariant mean on $L^{\infty}(G)$ such that $m\left(\mathbf{1}_{V}\right)=0$. This completes the proof.

\section{REFERENCES}

1. E. G. Effros, Property $\Gamma$ and inner amenability, Proc. Amer. Math. Soc. 47 (1975), 483-486. MR 50:8100

2. F. P. Greenleaf, Invariant means on topological groups and their applications, Van Nostrand, New York, 1969. MR 40:4776

3. A. T. M. Lau and A. L. T. Paterson, Operator theoretic characterizations of $[I N]$-groups and inner amenability, Proc. Amer. Math. Soc. 102 (1988), 893-897. MR 89f: 43003

4. _ Inner amenable locally compact groups, Trans. Amer. Math. Soc. 325 (1991), 155169. MR 91h: 43002

5. V. Losert and H. Rindler, Asymptotically central functions and invariant extensions of Dirac measure, Proc. Conf. Probability Measures on Groups VII (Oberwolfach, 1983), Lecture Notes in Math., vol. 1064, Springer, Berlin and New York, 1984, 368-378. MR 86e:43007

6. Conjugation-invariant means, Colloq. Math. 51 (1987), 221-225. MR 89d:43001

7. W. L. Paschke, Inner amenability and conjugation operators, Proc. Amer. Math. Soc. $\mathbf{7 1}$ (1978), 117-118. MR 57:13508

8. A. L. T. Paterson, Amenability, Math. Surveys Monographs, vol. 29, Amer. Math. Soc., Providence, RI, 1988. MR 90e:43001

9. J. P. Pier, Amenable locally compact groups, Wiley, New York, 1984. MR 86a:43001

10. C. K. Yuan, The existence of inner invariant means on $L^{\infty}(G)$, J. Math. Anal. Appl. 130 (1988), 514-524. MR 89g:43001

Department of Mathematics, Hokkaido University of Education, Hakodate, HachiMAN-CHO, HAKODATE, 040 JAPAN

E-mail address: ytakahas@hak.hokkyodai.ac.jp 